\title{
Semiclassical construction of random wave functions for confined systems
}

\author{
Juan Diego Urbina and Klaus Richter \\ Institut für Theoretische Physik, Universität Regensburg, 93040 Regensburg, Germany
}

(Received 3 September 2003; published 12 July 2004)

\begin{abstract}
We develop a statistical description of chaotic wave functions in closed systems obeying arbitrary boundary conditions by combining a semiclassical expression for the spatial two-point correlation function with a treatment of eigenfunctions as Gaussian random fields. Thereby we generalize Berry's isotropic random wave model by incorporating confinement effects through classical paths reflected at the boundaries. Our approach allows one to explicitly calculate highly nontrivial statistics, such as intensity distributions, in terms of usually few short orbits, depending on the energy window considered. We compare with numerical quantum results for the Africa billiard and derive nonisotropic random wave models for other prominent confinement geometries.
\end{abstract}

DOI: 10.1103/PhysRevE.70.015201

PACS number(s): 05.45.Mt, 03.65.Sq

In mesoscopic quantum systems many of the relevant physical phenomena can be described in the mean field approximation. In the semiclassical regime, characterized by (Fermi) wave lengths considerably smaller than the system size, challenges to theory are then posed owing to the arising complexity of the single-particle wave functions involved. In view of the correspondence principle their structures depend sensitively on phase space properties of the corresponding classical system [1]. This has called for an increasing theoretical investigation of statistical properties of eigenstates [2] since the seminal work by Berry [3]. This is of far more than theoretical interest as wave function fluctuations govern a variety of physical processes such as, e.g., photoabsoprtion of atoms and molecules or the measured conductance peaks statistics in quantum dots [4]. Wave function statistics entering into interaction matrix elements influence spectral properties of interacting quantum dots [5-8]. Scanning probe techniques and microwave experiments allow one to directly uncover the spatial structure of waves on mesoscopic scales [9].

Berry conjectured [3] that chaotic wave functions behave, with respect to their statistical properties, as Gaussian random fields, and arguments coming from semiclassics [3], quantum ergodicity [10], and information theory [11] support this Gaussian hypothesis. When supplemented with a Besseltype spatial two-point correlation function, the resulting theory is known as Berry's random wave model (RWM), since it is equivalent to consider the wave function as a random superposition of plane waves with locally fixed wave number magnitude. The RWM provides universal, systemindependent results consistent with random matrix theory (RMT). It constitutes the most widely used statistical description of chaotic eigenfunctions, as it has been extremely successful in predicting bulk or spatially averaged quantities. However, obviously, the RWM does not account for effects of confinement potentials which pose additional constraints to the wave functions, reducing their randomness, particularly in the spatial region close to the boundaries. This fact strongly diminishes the range of applicability of the usual RWM, since in many experiments the wave function near the boundary is particularly relevant (e.g., when measuring tunnel rates, the local density of states at surfaces or boundaries, or the conductance by attaching leads). Hence, very recently several papers appeared, where boundary effects have been incorporated into RWM approaches, however for specific geometries [12-15] only or in a qualitative way. [8]

We construct a RWM which allows one to incorporate effects of arbitrary confinements including Dirichlet-, Neumann-, and mixed boundary conditions in both billiard and smooth systems. To this end, we keep the Gaussian structure of the theory as presented in Refs. 3, 8, 17, and 18, but achieve a nonisotropic generalization by using a semiclassically exact expression for the two-point correlation function in terms of nondirect trajectories being reflected at the boundaries (extending an important previous work [18]). Both ingredients of our approach, the local Gaussian conjecture and the semiclassical two-point correlation, will be numerically tested and confirmed.

Defining the ensemble. We focus on two-dimensional clean, closed systems with time reversal symmetry [19]. We consider energy averages over a set of $N_{W}$ normalized solutions $\psi_{n}(\vec{r})$ of the Schrödinger equation with nondegenerate energies $E_{n}$ lying inside an interval $W=[e-\delta e / 2, e+\delta e / 2]$. In Thomas-Fermi approximation, $N_{W}=(2 \pi \hbar)^{-2} \delta e \Omega(e)$ with $\Omega(e)$ the volume of the energy shell. We assume $\delta e / e \ll 1$, which is always achieved in the semiclassical limit we are interested in. Considering such energy averages is standard for disorder-free mesoscopic systems as they allows for random matrix approaches [20]. Moreover, experiments often involve averages over finite energy windows. In particular, the averaged eigenfunction intensity to be considered is proportional to the local density of states, relevant to many experiments such as scanning probe, quantum transport, photoabsorption and ionization measurements.

At a fixed position $\vec{r}=(x, y)$ we will probe wave function amplitudes by means of a function $F\left(u_{n}\right)=F\left(\psi_{n}(\vec{r})\right)$ which fluctuates when varying $E_{n}$ and the corresponding state $\psi_{n}$ inside $W$. We define the spectral average of $F$ at $\vec{r}$ as $\mathcal{F}(\vec{r})$ $\equiv 1 / N_{W} \Sigma_{E_{n} \in W} F\left(\psi_{n}(\vec{r})\right)$. An important example is the distribution of intensities

$$
I(w ; \vec{r}) \equiv \frac{1}{N_{W}} \sum_{E_{n} \in W} \delta\left(w-\left|\psi_{n}(\vec{r})\right|^{2}\right) .
$$

This is easily generalized to higher-order statistics such as the spatial correlation intensities, $Y\left(w_{1}, w_{2} ; \vec{r}_{1}, \vec{r}_{2}\right)$ 
$\equiv 1 / N_{W} \Sigma_{E_{n} \in W} \delta\left(w_{1}-\left|\psi_{n}\left(\vec{r}_{1}\right)\right|^{2}\right) \delta\left(w_{2}-\left|\psi_{n}\left(\vec{r}_{2}\right)\right|^{2}\right)$, and to functions $F(\vec{u})$ depending not only on the eigenfunctions but also on their derivates of any order

$$
\mathcal{F}\left(\vec{r}_{1}, \ldots, \vec{r}_{M}\right) \equiv \frac{1}{N_{W}} \sum_{E_{n} \in W} F\left(u_{n}^{1}\left(\vec{r}_{1}\right), \ldots, u_{n}^{M}\left(\vec{r}_{M}\right)\right),
$$

where $u_{n}^{\alpha}\left(\vec{r}_{i}\right)=\partial_{x_{i}}^{l} \partial_{y_{i}}^{m_{\alpha}} \psi_{n}\left(x_{i}, y_{i}\right)$ with integers $l_{\alpha}, m_{\alpha}$. If there are $J$ different positions among the set $\vec{r}_{1}, \ldots, \vec{r}_{M}$, we call $\mathcal{F}\left(\vec{r}_{1}, \ldots, \vec{r}_{M}\right)$ a $J$-point statistics. In this paper, a central quantity is the two-point correlation function

$$
R\left(\vec{r}_{i}, \vec{r}_{j}\right) \equiv \frac{1}{N_{W}} \sum_{E_{n} \in W} \psi_{n}\left(\vec{r}_{i}\right) \psi_{n}\left(\vec{r}_{j}\right)
$$

since the average of any expression bilinear in the wave function can be expressed through this correlation.

The local Gaussian conjecture. Using the exact probability distribution $P(\vec{u})=1 / N_{W} \Sigma_{E_{n} \in W} \delta\left(\vec{u}-\vec{u}_{n}\right)$ the statistics (2) can be calculated as $\mathcal{F}\left(\vec{r}_{1}, \ldots, \vec{r}_{M}\right)=\int_{-\infty}^{\infty} F(\vec{u}) P(\vec{u}) d \vec{u}$. Instead, the local Gaussian conjecture for the statistics of eigenfunctions of classically chaotic quantum systems claims that the energy ensemble is described as a Gaussian stationary process. More precisely, this means to assume (in the weak sense) $P(\vec{u})=P^{G}(\vec{u})$ with

$$
P^{G}(\vec{u})=(2 \pi)^{-M / 2}(\operatorname{det} \mathbf{C})^{-1 / 2} \exp \left(-\frac{1}{2} \vec{u}\left(\mathbf{C}^{-1}\right) \vec{u}\right),
$$

where the correlation matrix $\mathbf{C}=\mathbf{C}\left(\vec{r}_{1}, \ldots, \vec{r}_{J}\right)$ has entries $c_{\alpha, \beta}=1 / N_{W} \Sigma_{E_{n} \in W} u_{n}^{\alpha} u_{n}^{\beta}$. Since all these entries consist of averages over quantities bilinear in the eigenfunctions, the knowledge of the two-point correlation function (3) completely determines, under the Gaussian assumption, the matrix $\mathbf{C}$ and all statistical properties. Versions of this conjecture have also been used to describe scarring effects [2] and tunneling rates [11].

Applying this approach to the intensity distribution $I(w ; \vec{r})$, the matrix $\mathbf{C}$ reduces to a single entry $c_{1,1}=R(\vec{r}, \vec{r})$. Using the above expression for $P(u)$ we find

$$
I^{G}(w ; \vec{r})=\frac{1}{\sqrt{2 \pi w R(\vec{r}, \vec{r})}} \exp \left(-\frac{w}{2 R(\vec{r}, \vec{r})}\right) .
$$

Due to the boundary, $R(\vec{r}, \vec{r})$ will depend on $\vec{r}$ (as will be discussed in Fig. 2). This constitutes a nonisotropic generalization of the (isotropic) Porter-Thomas distribution, given by $R(\vec{r}, \vec{r})=1 / A$ for a billiard of area $A$.

The intensity correlation, $Y\left(w_{1}, w_{2}, \vec{r}_{1}, \vec{r}_{2}\right)$, involves a 2 $\times 2$ matrix with elements $c_{i, j}=R\left(\vec{r}_{i}, \vec{r}_{j}\right)$. The Gaussian integrals then give the nonisotropic generalization

$$
\begin{aligned}
Y^{G}\left(w_{1}, w_{2} ; \vec{r}_{1}, \vec{r}_{2}\right)= & \frac{1}{2 \pi \sqrt{w_{1} w_{2} \operatorname{det} \mathbf{C}}} \cosh \left(\frac{\sqrt{w_{1} w_{2}} c_{1,2}}{\operatorname{det} \mathbf{C}}\right) \\
& \times \exp \left(-\frac{c_{1,1} w_{2}+c_{2,2} w_{1}}{2 \operatorname{det} \mathbf{C}}\right)
\end{aligned}
$$

of the distribution studied in [16,17].

To check our main assumption, the position-dependent Gaussian conjecture (4), for a generic chaotic system, we have computed the integrated intensity distribution $P(w)$ $=\int I(w ; \vec{r}) d \vec{r}$ based on numerical results [21] for the kicked

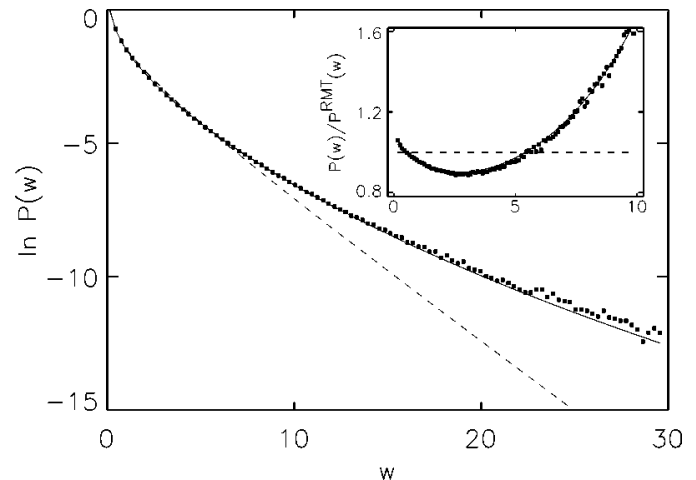

FIG. 1. Integrated distribution $P(w)=\int I(w ; \vec{r}) d \vec{r}$. The symbols are exact quantum results for the kicked rotor using Eq. (1), the dashed line is the Porter-Thomas distribution $P^{\mathrm{RMT}}(w)$ and the solid line is $\int I^{G}(w ; \vec{r}) d \vec{r}$ with $I^{G}(w, \vec{r})$ from Eq. (4) and position dependent correlation (3) obtained from quantum mechanical numerical results for the wave functions. The local Gaussian assumption adequately describes the bulk (inset) and tails beyond the RMT result.

rotator. As shown in Fig. 1 the exact calculations (symbols) show distinct deviations from the Porter-Thomas distribution $P^{\mathrm{RMT}}(A w)=(2 \pi w)^{-1 / 2} e^{-w / 2}$ (dashed line) both in the tails and body (inset). If we use instead $\int I^{G}(w ; \vec{r}) d \vec{r}$ with the local distribution (4) and use the numerical values of the wave functions to calculate the correlation (3) the agreement is impressive (solid line). That is, if we use the numerically exact two-point correlation function, deviations from universality [22] are perfectly incorporated in the Gaussian theory through spatial fluctuations of the correlation matrix.

Semiclassical construction of the correlation matrix. The above scheme critically depends on how precisely $R\left(\vec{r}_{i}, \vec{r}_{j}\right)$ can be calculated [23]. This is a serious issue in the theory of chaotic quantum systems where no analytical expressions for the eigenfunctions exist, and approximate methods are required. It turns out tobe convenient to express $R\left(\vec{r}_{i}, \vec{r}_{j}\right)$ through the Green function $G\left(\vec{r}_{i}, \vec{r}_{j} ; E+i 0^{+}\right)$

$$
R\left(\vec{r}_{i}, \vec{r}_{j}\right)=\frac{1}{\pi} \frac{1}{N_{W}} \int_{e-\delta e / 2}^{e+\delta e / 2} \operatorname{Im} G\left(\vec{r}_{i}, \vec{r}_{j} ; E+i 0^{+}\right) d E
$$

since a variety of approximations exists for $G$.

We start from the the exact multiple reflection expansion of the Green function [24] and consider the two leading terms, $G \simeq G^{(0)}+G^{(1)}$, to calculate $R\left(\vec{r}_{i}, \vec{r}_{j}\right)$. Here $G^{(1)}$ represents all quantum paths between $\vec{r}_{i}$ and $\vec{r}_{j}$ hitting the boundary once (including nonspecular reflections) and $G^{(0)}$ denotes the contribution from the direct path joining $\vec{r}_{i}$ and $\vec{r}_{j}$. The corresponding isotropic contribution $R^{\mathrm{is}}\left(\vec{r}_{i}, \vec{r}_{j}\right)$ to $R$ is obtained by means of the short-time propagator. For small distances $q=\left|\vec{r}_{i}-\vec{r}_{j}\right|[25]$ it is evaluated at the mean potential $V(\vec{Q})$ for a local wave number $\hbar k=[2 m(e-V(\vec{Q}))]^{1 / 2}$ with mass $m$ and $\vec{Q}=\left(\vec{r}_{i}+\vec{r}_{j}\right) / 2$ as $G^{(0)}=-i m /\left(2 \hbar^{2}\right)\left(J_{0}^{+}(k q)\right.$ $\left.+i Y_{0}(k q)\right)\left(J_{0}, Y_{0}\right.$ the Bessel and modified Bessel functions). For $q \rightarrow 0$ Eq. (6) then gives Berry's result 


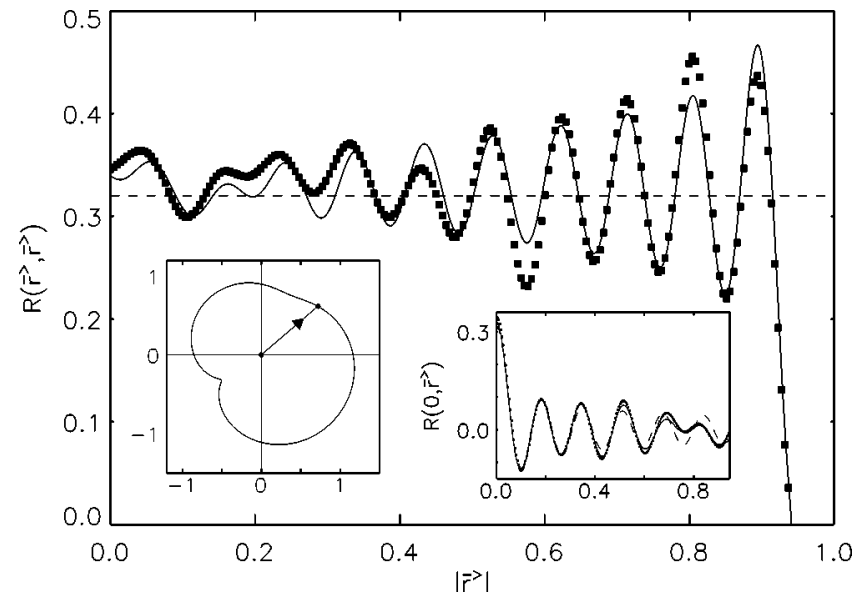

FIG. 2. Two-point correlation function $R(\vec{r}, \vec{r})$ and $R(0, \vec{r})$ (right inset) for $\vec{r}$ pointing along the line indicated in the Africa billiard (left inset). The symbols mark numerical quantum results for $R$, Eq. (3) [30]. The thin lines depict the semiquantum prediction employing Eq. (6) where the Green function is approximated by a sum over paths, including diffraction effects, with at most one reflection at the boundary. The dashed lines show the isotropic RWM result (7).

$$
R^{i s}\left(\vec{r}_{i}, \vec{r}_{j}\right)=[2 \pi m / \Omega(e)] J_{0}(k q) .
$$

It can be obtained using a number of equivalent considerations $[3,18,26,10]$ and defines the isotropic RWM.

The power of the representation (6) for $R\left(\vec{r}_{i}, \vec{r}_{j}\right)$ combined with the Green function expansion is demonstrated for the Africa billiard [28] (left inset of Fig. 2). The numerical evaluation of $R(\vec{r}, \vec{r})$ (Fig. 2) and $R(0, \vec{r})$ (right inset in Fig. 2) within this approximation is extremely fast and the results (thin lines) show considerable agreement with numerically exact, but time consuming quantum mechanical reference calculations (symbols). The boundary effects [e.g., the oscillations in $R(\vec{r}, \vec{r})]$ are adequately incorporated in the onebounce treatment, but evidently beyond the isotropic RWM [Eq. (7), dashed lines] which yields in this case $R(\vec{r}, \vec{r})$ $=1 / \pi$.

In the semiclassical limit the terms in the multiple reflection expansion can be further approximated by the respective semiclassical Green function [1] $G^{\mathrm{sc}}\left(\vec{r}_{i}, \vec{r}_{j} ; E\right)$ $=(i \hbar \sqrt{2 \pi i \hbar})^{-1} \Sigma_{\gamma}\left|D_{\gamma}\right|^{1 / 2} \exp \left(i / \hbar S_{\gamma}-i \mu_{\gamma} \pi / 2\right)$ where $\gamma$ now labels the classical paths joining $\vec{r}_{i}$ with $\vec{r}_{j}$. D $D_{\gamma}$ and $\mu_{\gamma}$ are classical quantities slowly varying with energy, and $S_{\gamma}\left(\vec{r}_{i}, \vec{r}_{j} ; E\right)=\int_{\gamma} \vec{p} \cdot d \vec{q}$ is the classical action along the path. For energy windows with $\delta e / e \ll 1$ the action can be expanded as $S_{\gamma}(E) \simeq S_{\gamma}(e)+T_{\gamma} \delta e / 2$. The energy integral (6) then yields the two-point correlation function

$$
\begin{aligned}
R\left(\vec{r}_{i}, \vec{r}_{j}\right) \simeq & \frac{2 m}{\Omega(e)} \sum_{\gamma} \Gamma\left(\frac{T_{\gamma}}{\tau_{W}}\right)\left|2 \pi \hbar D_{\gamma}\right|^{1 / 2} \\
& \times \cos \left[S_{\gamma}\left(\vec{r}_{i}, \vec{r}_{j} ; e\right) / \hbar-\mu_{\gamma} \pi / 2\right]
\end{aligned}
$$

in terms of classical paths. In Eq. (8), $T_{\gamma}$ is the traversal time of path $\gamma, \Gamma(x)=\sin (x) / x$ and $\tau_{W}=2 \hbar / \delta e$ is a characteristic cutoff time associated with the window $W$. Equation (8) is similar to the correlation derived for disordered systems and conjectured to be valid also for the ballistic case [18] which corresponds to the case $\Gamma(x)=1$. Our result including the damping function is, however, conceptually superior for both practical and theoretical reasons: In practice $\tau_{W}$ controls the maximum orbit length to be considered which otherwise must be artificially set to the Heisenberg time, an extremely long time in classical terms that makes explicit calculations hopeless. Theoretically, $\Gamma(x)$ makes the result (8) compatible with the definition (3) [29] which is essential to cope with the normalization problem [27].

The use of $R^{\text {is }},(7)$, is adequate for an additional spatial average, since for fixed $\vec{Q}$ the integration over the relative position $\vec{q}$ in any small region will contain the continuous set of paths joining $\vec{r}_{i}$ with $\vec{r}_{i}+\vec{q}$ directly and the contribution from nondirect paths isolated in chaotic systems. Hence in the semiclassical limit the spatial integration over the continuous set of direct paths yields the isotropic result as the dominant contribution.

On the contrary, for a pure energy average the contribution to $R$ from nondirect paths is of the same semiclassical order as that from direct paths. However, the window size $\delta e$ determines the maximum length of the nondirect paths contributing to the correlation function. The major step beyond the isotropic case is to consider an energy window such that only the direct and shortest nondirect paths significantly contribute to $R$, which is also particularly experimentally relevant.

To this end we specify the nondirect paths more precisely. In billiards the first nondirect contribution is given by a sum $\sum_{p} R^{(p)}\left(\vec{r}_{i}, \vec{r}_{j}\right)$ over usually few classical trajectories $p$ hitting the boundary once. For given initial and final positions $\vec{r}_{i}, \vec{r}_{j}$ each one-bounce path $p$ is uniquely characterized by the position $\vec{r}_{p}$ where it is reflected. The path length is $L_{p}=L_{i p}$ $+L_{j p}$ with $L_{i p}=\left|\vec{r}_{i}-\vec{r}_{p}\right|, L_{j p}=\left|\vec{r}_{j}-\vec{r}_{p}\right|$. Denoting by $\kappa_{p}$ and $\theta_{p}$ the local boundary curvature and reflection angle at $\vec{r}_{p}$, a simple calculation yields

$$
\begin{aligned}
R^{(p)}\left(\vec{r}_{i}, \vec{r}_{j}\right) \simeq & \Gamma\left(\frac{k L_{p} \delta e}{e}\right)\left|2 \kappa_{p}\left(\frac{L_{i p} L_{j p}}{L_{p} \cos \theta_{p}}\right)-1\right|^{-1 / 2} \\
& \times \frac{1}{A \sqrt{2 \pi k L_{p}}} \cos \left(k L_{p}-\frac{\pi}{4}+\phi_{p}\right) .
\end{aligned}
$$

Here $\phi_{p}$ takes into account the boundary conditions at the reflection point, as given, e.g., in Ref. 31.

$R\left(\vec{r}_{i}, \vec{r}_{j}\right)=R^{\text {is }}\left(\vec{r}_{i}, \vec{r}_{j}\right)+\sum_{p} R^{(p)}\left(\vec{r}_{i}, \vec{r}_{j}\right)$, together with the semiclassical expressions (7) and (9), provide the entries for the correlation matrix $\mathbf{C}$, from which arbitrary statistical measures [such as $I,(1)$, and $Y,(5)]$ for the wave functions can be deduced for the nonisotropic case. Moreover, this semiclassical correlation yields closed analytical expressions for statistical quantities for chaotic systems such as the stadium-, cardiod-, or Sinai billiard, since in these cases all the parameters required are readily calculated from geometrical considerations.

The approach is easily generalized to include AharonovBohm flux lines or smooth boundary potentials.

Microscopic derivation of nonisotropic random wave models. We demonstrate the power of the semiclassical 
scheme outlined above by computing further wave function correlators for selected, representative examples. First we show the role of the confinement by considering points $\vec{r}$ close to the billard boundary. In [12] this is treated by approximating the boundary by an infinite straight line $y=y_{0}$ and considering an ensemble of random superpositions of plane waves $\psi^{r}(\vec{r})$ satisfying the mixed boundary condition $\left.\left(\partial_{y} \psi^{r}(\vec{r}) \sin a+k \psi^{r}(\vec{r}) \cos a\right)\right|_{y=y_{0}}=0$. Here $a$ is a positiondependent parameter and $k$ the local wave number. By ensemble average a variety of two-point correlations was derived in Ref. [12] and used to calculate specific statistical observables. To illustrate our method and for the sake of comparison we consider just one such average, namely $\left\langle\psi^{r}(\vec{r}) \partial_{y} \psi^{r}(\vec{r})\right\rangle$. In terms of the two-point correlation function it reads $\left\langle\psi(\vec{r}) \partial_{y} \psi(\vec{r})\right\rangle=1 /\left.2\left(\partial_{y_{i}}+\partial_{y_{j}}\right) R\left(\vec{r}_{i}, \vec{r}_{j}\right)\right|_{y_{i}=y_{j}=y}$. Close to the boundary only the direct and the shortest nondirect path contribute. For mixed boundary conditions the extra phase $\phi_{p}$ in Eq. (9) is given semiclassically [31] by $\phi_{p}=\pi$ $-2 \arctan \left(\tan a \cos \theta_{p}\right)$. Substitution into Eq. (9) gives the approximate correlation function close to an arbitrary boundary. To leading order in $k$ we get

$$
\begin{aligned}
\left\langle\psi(\vec{r}) \partial_{y} \psi(\vec{r})\right\rangle= & \Gamma\left(\frac{2 k d(\vec{r}) \delta e}{e}\right) \frac{1}{\sqrt{|1-\kappa d(\vec{r})|}} \frac{k}{A} \frac{1}{\sqrt{\pi k d(\vec{r})}} \\
& \times \sin \left(2 k d(\vec{r})-2 a-\frac{\pi}{4}\right),
\end{aligned}
$$

where $d(\vec{r})$ is the distance from $\vec{r}$ to the boundary. For flat boundaries or very short $d(\vec{r})$ the semiclassical results are the large $k$ limits of the integral expressions given in Ref. 12. The Dirichlet and Neumann cases studied in Ref. 13 are particular cases of Eq. (10) with $a=0$ and $a=\pi / 2$. The results for a wedge [15] with opening $\pi / n$ ( $n$ integer) is also derived in the present scheme by taking the shortest $n$ classical paths hitting alternatively the edges of the wedge. We also recover the large $k$ form of the correlation function [14] for an infinite potential ramp, using only one classical trajectory. Our derivation of these correlation functions does not involve any statistical assumption.

To summarize, we showed how to efficiently treat wave function statistics for closed systems by merging statistical with semiclassical concepts. We demonstrated that all known (to us) results [12-15] for specific, nonisotropic random wave models are particular cases of the general approach presented here. It provides closed analytical expressions for statistical measures in terms of geometrical quantities and builds the framework for incorporating arbitrary boundary conditions and confinement geometries.

We thank S. Gnutzmann, G. Foltin, P. Schlagheck, M. Sieber, U. Smilansky, and M. Turek for helpful conversations and A. Ossipov and T. Kottos for providing their numerical results for the kicked rotor. This work was supported by the Graduiertenkolleg "Nonlinearity and Nonequilibrium in Condensed Matter" of the Deutsche Forschungsgemeinschaft.
[1] M. Gutzwiller, Chaos in Classical and Quantum Mechanics (Springer, New York, 1990).

[2] See, e.g., the recent reviews by Kaplan and Heller, Fishman, and Mirlin in Supersymmetry and Trace Formulae, edited by I. V. Lerner et al. (Kluwer, New York, 1999), and references therein.

[3] M. V. Berry, J. Phys. A 10, 2083 (1977).

[4] A. M. Chang et al., Phys. Rev. Lett. 76, 1695 (1996); J. A. Folk et al., ibid. 76, 1699 (1996).

[5] K.-H. Ahn et al., Phys. Rev. Lett. 83, 4144 (1999).

[6] Y. Alhassid, Rev. Mod. Phys. 72, 895 (2000).

[7] A. D. Mirlin, Phys. Rep. 326, 259 (2000).

[8] D. Ullmo et al., Phys. Rev. B 64, 245324 (2001).

[9] See, e.g., Y. H. Kim, M. Barth, U. Kuhl, and H. J. Stöckmann, e-print cond-mat/0301411, and references therein.

[10] G. Blum et al., Phys. Rev. Lett. 88, 114101 (2002).

[11] E. E. Narimanov et al., Phys. Rev. B 64, 235329 (2001).

[12] M. V. Berry and H. Ishio, J. Phys. A 35, 5961 (2002).

[13] M. V. Berry, J. Phys. A 35, 3025 (2002).

[14] W. E. Bies and E. J. Heller, J. Phys. A 35, 5673 (2002).

[15] W. E. Bies et al., J. Phys. A 36, 1605 (2003).

[16] V. N. Prigodin, Phys. Rev. Lett. 75, 2392 (1995).

[17] M. Srednicki, Phys. Rev. E 54, 954 (1996).
[18] S. Hortikar and M. Srednicki, Phys. Rev. Lett. 80, 1646 (1998).

[19] The generalization to arbitrary dimensions and systems with broken time reversal symmetry is straightforward.

[20] C. W. J. Beenakker, Rev. Mod. Phys. 69, 731 (1997).

[21] A. Ossipov et al., Phys. Rev. E 65, 055209 (2002).

[22] In this case due to pre-localized states. Similar effects are found in billiard systems due to scarring phenomena [23]; see L. Kaplan, Nonlinearity 12, R1 (1999); see also Ref. [2] and references therein.

[23] E. J. Heller Phys. Rev. Lett. 53, 1515 (1984).

[24] R. Balian and C. Bloch, Ann. Phys. (N.Y.) 60, 401 (1970).

[25] A precise criterium is $\left|\vec{r}_{i}-\vec{r}_{j}\right| \ll a\left(\vec{r}_{i}+\vec{r}_{j}\right) / 2$ with $[a(\vec{r})]^{-1}$ $=\nabla \log |V(\vec{r})|$.

[26] F. Toscano et al., Phys. Rev. E 65, 036201 (2002).

[27] J. D. Urbina and K. Richter, J. Phys. A 36, L495 (2003).

[28] M. Robnik, J. Phys. A 17, 1049 (1984).

[29] $R$ must satisfy $N_{W} \int R(\vec{r}, \vec{r}) R\left(\vec{r}, \vec{r}_{j}\right) d \vec{r}=R\left(\vec{r}_{i}, \vec{r}_{j}\right)$ for consistency; this requires a window function.

[30] Quantum results for an energy interval $\left[E_{330}, E_{370}\right]$ and mean level spacing 4 (for billiard area $A=\pi$ ).

[31] M. Sieber et al., J. Phys. A 28, 5041 (1995). 